\title{
NUMERICAL SIMULATION OF REYNOLDS NUMBER EFFECT OF A FREE INCOMPRESSIBLE ISOTHERMAL TURBULENT COAXIAL JET
}

\author{
Olanrewaju Miracle Oyewola $\bowtie$ \\ School of Mechanical Engineering \\ Fiji National University \\ Ratu Mara road, Samabula, Suva, Fiji, 3722 \\ Department of Mechanical Engineering ${ }^{1}$ \\ Olanrewaju.oyewola@fnu.ac.fj,oooyewola001@gmail.com \\ Olawale Saheed Ismail \\ Department of Mechanical Engineering ${ }^{l}$ \\ Lateef Anjola Sanni \\ Department of Mechanical Engineering ${ }^{l}$ \\ ${ }^{1}$ University of Ibadan \\ Sango-Ojo road, Ibadan, Oyo State, Nigeria, 200284
}

$\triangle$ Corresponding author

\begin{abstract}
This paper studies the effect of Reynolds number on a two-dimensional free incompressible isothermal coaxial turbulent jet over a range of high Reynolds numbers. This is necessary because of its application in noise control and mixing. The Reynolds numbers at the nozzle exit were 9824, 19648, 29472, 39296 and 49120. The models were designed in ANSYS Design Modeler and the numerical simulation was done using a finite volume based Computational Fluid Dynamics (CFD) in ANSYS FLUENT using the two-dimensional Realizable turbulence model. The Governing equations were discretized using the finite volume method with the solution based on the PISO algorithm. The decay of centerline velocity, turbulent kinetic energy profile, the radial profile of axial velocity and similarity profile were investigated along the flow direction. Contour plot indicates that the velocity is high at the jet exit and decreases downstream due to the rapid mixing of the inner and outer jet and the surrounding fluid. It is found generally that Reynolds number plays significant role especially before self-similarity region. The result shows that increasing the Reynolds number give rise to more turbulence which in turn decreases the potential core length, turbulent kinetic energy and enhances the mixing of the fluid. However, at the jet exit, the flow with the lowest Reynolds number has the highest turbulent kinetic energy because it suffers the greater shear. The spreading of the jet was more or less independent of the Reynolds number beyond the self-similarity region. It is also found that the velocity profile is brought to congruence at about $z / D=25$ for the Reynolds numbers considered.

Keywords: Co-axial jet, Numerical simulation, Entrainment, turbulence modeling, Reynold number, PISO.
\end{abstract}

DOI: $10.21303 / 2461-4262.2022 .001896$

\section{Introduction}

The study of the fluid behavior of a coaxial jet has been a subject of interest in the last two decades [1] due to their potential to reduce noise, improve combustion and enhance mixing. The mixing capability of the coaxial jet is influenced by the jet exit conditions such as the velocity ratio, diameter ratio, and the Reynolds number.

The Reynolds number which is a function of the velocity ratio and the diameter ratio of the system plays a crucial role in determining the turbulent characteristics of the jet. This has a direct effect on the mixing process of the coaxial jet. It is therefore important to understand the role of the Reynolds number on jet development.

A review of the literature that deals with the factors affecting the fluid dynamics of the coaxial jet showed that literature is sparse to some degree on the Reynolds number effect especially on jet development over a range of high Reynolds numbers. However, some of the work has focussed on low Reynolds numbers where serious mixing was not involved. For instance, [2] 
numerically studied the effect of velocity ratios between the inner and outer streams of the jet on the coaxial jet flow field using the Reynolds Averaged Navier-Stokes (RANS) turbulence model. It was observed that for the different velocity ratios there was a variation in the decay of the mean longitudinal velocities along the jet centerline, mean and fluctuating velocities. It was also shown that the coaxial jet flow field did not show self-similarity up to $25 D_{i}$ (where $D_{i}$ is the inner diameter of the annulus pipe).

Moreover, [3] investigated the stirring and mixing properties of one phase coaxial jet, with large outer to inner velocity ratio. It was suggested that the only two quantities necessary to understand the rate of interface corrugation, the time of onset of molecular mixing and the subsequent rate of change of the medium composition are the initial vorticity thickness $\delta$, set by the initial Reynolds number Re, and the elongation rate:

$$
\gamma=\left(\frac{u_{2}}{e}\right)\left(1-\frac{1}{r_{u}}\right)
$$

constructed with the net velocity difference between the streams and the gap width e. Interestingly, [4] used ANSYS CFX algorithm solver to solve RANS and SST model equations in simulating the interrelation of fluid mechanics of formation of co-axial jets and the development downstream of the jet emergence for Reynolds numbers of 10000, 20000, and 50000 based on diameter ratio in the turbulent flow simulation. Their results showed that at an appropriate downstream length, the width of the jet was recorded to be independent of both the geometrical parameters and the Reynolds number. However, the scope of their work was limited.

It should be noted that [5] conducted a Large Eddy Simulation (LES) study of mixing and intermittency of a coaxial turbulent jet discharging into an unconfined domain. The coaxial jet considered has relatively high jet velocities for both core and annular jets with an aspect ratio (core jet to annular jet) of 1.48 . The passive scalar fields developing from the core and bypass flow were found to exhibit differences at near and far field locations. The calculated velocity fluctuations resulted in intermittent behaviour near the jet boundaries for both velocity and passive scalar fields. Despite interesting results emanated from their work, the influence of Reynolds number was not considered. Further, [1] in their work titled «CFD Analysis of Supersonic Coaxial Jets on Effect of Spreading Rates» designed and analyzed two-dimensional coaxial jet profiles of different area ratios $(0.9,1.8,2.9$ and 4.3$)$. Two-dimensional density based energy equation and $k-\varepsilon$ turbulence model with a primary supersonic flow and secondary subsonic flow were solved. It was observed that increasing the area ratio results in less turbulence which in turn increases the potential core length, acoustics power level, turbulent kinetic energy and generates more noise.

In spite of considerable previous work from numerical and experimental point of views on coaxial turbulent jets, there are still some important aspects which require greater attention. For instance, it is important to establish the effects of Reynolds number on jet development over a range of high Reynolds number. Such establishment is essential to gain more insight to their mixing characteristics, particularly relevant to engineering applications. To satisfy this need, the objective of this study is to examine numerically the effect of the jet exit Reynolds number on the flow field of the turbulent coaxial jet.

\section{Materials and methods}

\section{1. Mathematical modelling}

The objective of this study is to investigate the behaviour of the effect of Reynolds number on the coaxial jet flow field. For this purpose, the Realizable model was used. This model is a two - equation model based on the eddy viscosity hypothesis.

The governing equation in the cylindrical coordinate system is expressed in (1) and Table $\mathbf{1}$ in the general form:

$$
\frac{\partial}{\partial t}(\rho \varphi)+\frac{\partial}{\partial z}(\rho v \varphi)+\frac{1}{r} \frac{\partial}{\partial z}(\rho r u \varphi)=\frac{\partial}{\partial z}\left(\Gamma_{\varphi} \frac{\partial \varphi}{\partial z}\right)+\frac{1}{r} \frac{\partial}{\partial r}\left(\Gamma_{\varphi} r \frac{\partial \varphi}{\partial z}\right)+S_{\varphi},
$$




$$
\begin{gathered}
P_{k}=\mu_{t} \frac{\partial u}{\partial r}\left(\frac{\partial u}{\partial r}+\frac{\partial v}{\partial r}\right), \mu_{t}=\rho C_{\mu} \frac{k^{2}}{\varepsilon}, C_{\mu}=\frac{1}{A_{0}+\frac{A_{s} k u^{*}}{\varepsilon},} \\
U^{*}=\sqrt{S_{i j} S_{i j}+\tilde{\Omega}_{i j} \tilde{\Omega}_{i j}}, \Omega_{i j}=\tilde{\Omega}_{i j}-\varepsilon_{i j k} \omega_{k}, \\
A_{0}=4.04, A_{s}=6^{0.5} \cos \varphi, \varphi=\frac{1}{3} \cos ^{-1}(\sqrt{6} W), \\
W=\frac{S_{i j} S_{j i} S_{k l}}{\widetilde{S}}, \tilde{S}=\sqrt{S_{i j} S_{i j}}, S_{i j}=\frac{1}{2}\left(\frac{\partial u_{j}}{\partial x_{i}}+\frac{\partial u_{i}}{\partial x_{j}}\right), \\
C_{\varepsilon 1}=1.45, C_{\varepsilon 2}=1.9, \sigma_{k}=1.0, \text { and } \sigma_{\varepsilon}=1.2,
\end{gathered}
$$

where $v$ and $u$ are the time-averaged velocities in the axial and radial directions, respectively; $P_{k}$ is the turbulent production term; $P$ is the Reynolds averaged pressure; $\sigma_{k}, \sigma_{\varepsilon}, C_{\varepsilon 1}$ and $C_{\varepsilon 2}$ are the turbulent model constants; $r$ is the radial distance of the jet; $z$ is the axial distance measured downstream of the jet exit; $S_{i j}$ is the mean strain rate tensor; $\varepsilon$ is the turbulent dissipation rate; $\mu$ is the dynamic viscosity; $\rho$ is the density; $k$ is the turbulent kinetic energy and $\varphi$ is the various time-averaged quantities.

\section{Table 1}

The Transport Equation and the model constants

\begin{tabular}{lccc}
\hline \multicolumn{1}{c}{ Equation } & $\boldsymbol{\varphi}$ & $\boldsymbol{\Gamma}_{\boldsymbol{\varphi}}$ & $\boldsymbol{S}_{\boldsymbol{\varphi}}$ \\
\hline Continuity & 1 & 0 & 0 \\
$z$-momentum & $v$ & $\mu+\mu_{t}$ & $\frac{\partial}{\partial z}\left(\left(\mu+\mu_{t}\right) \frac{\partial v}{\partial z}\right)+\frac{1}{r} \frac{\partial}{\partial r}\left(r\left(\mu+\mu_{t}\right) \frac{\partial u}{\partial z}\right)-\frac{\partial P}{\partial z}$ \\
$r$-momentum & $u$ & $\mu+\mu_{t}$ & - \\
Kinetic Energy & $k$ & $\mu+\frac{\mu_{t}}{\sigma_{k}}$ & - \\
Dissipation Rate & $\varepsilon$ & $\mu+\frac{\mu_{t}}{\sigma_{k}}$ & $P_{k}-\rho C_{1} S_{c}-\rho C_{2} \frac{\varepsilon^{2}}{k+\sqrt{v \varepsilon}} S_{c} \frac{\varepsilon}{k}\left(C_{\varepsilon 1} P_{k}-C_{\varepsilon 2} \rho \varepsilon\right)$
\end{tabular}

The transport equations and the constants of the $k-\varepsilon$ turbulence model of [6] used for the present computations are summarized in Table 1. In addition, $\Gamma_{\varphi}$ and $S_{\varphi}$ for various conservation equations are also given in the Table $\mathbf{1 .}$

\section{2. Solution domain and boundary conditions}

The flow field of the coaxial jet was solved as a two-dimensional axisymmetric flow, using finite volume method. Due to the symmetric nature of the problem, the solution domain is represented with one-half of the domain as shown in Fig. 1.

In order to get rid of the effects of the exit boundary conditions on the flow, the dimension of the solution domain was stretched to $100 D_{o}$ and $40 D_{o}$. The imposed boundary conditions include the assumption of a constant pressure and velocities perpendicular to the surfaces. The standard atmospheric pressure of $101.325 \mathrm{~Pa}$ and a gauge pressure of 0 were applied to the outer boundary. The Reynolds number based on the fluid properties, velocity ratio $(\lambda)$ and diameter ratio of the system was varied as 9824, 19648, 29472, 39296 and 49120 as shown in Table 2 using (2):

$$
\operatorname{Re}=\frac{d_{o} u_{o}}{v}\left[1+\frac{1-\lambda^{-1}}{\lambda^{-1}\left(\frac{d_{o}}{d_{i}}\right)^{2}}\right]^{1 / 2}
$$




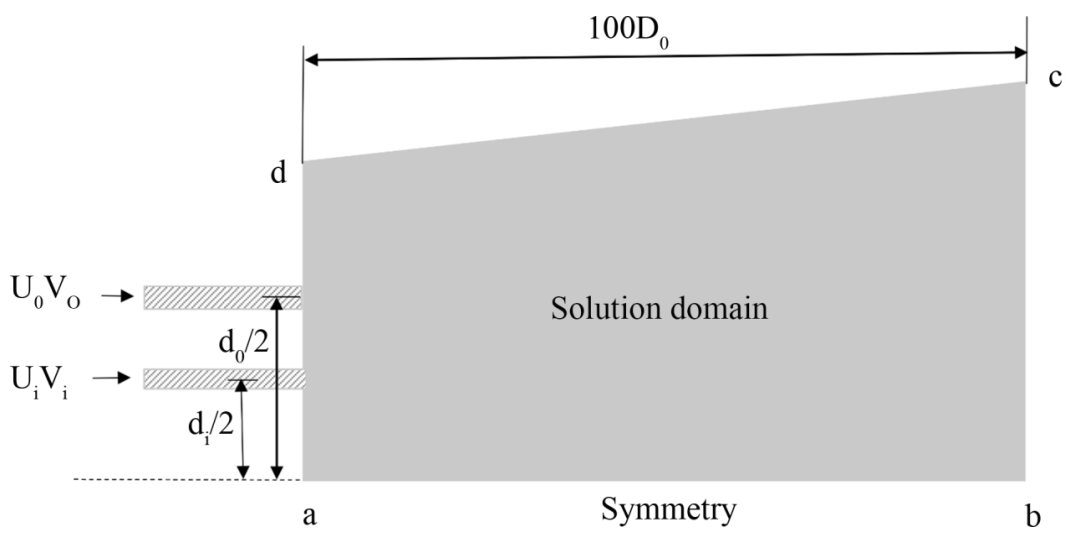

Fig. 1. The Solution domain

Table 2

The Flow Settings

\begin{tabular}{ccccc}
\hline $\boldsymbol{u}_{\boldsymbol{i}}\left(\mathbf{m} \mathbf{s}^{(-\mathbf{1})}\right)$ & $\boldsymbol{d}_{\boldsymbol{i}}(\mathbf{m})$ & $\boldsymbol{u}_{\boldsymbol{o}}\left(\mathbf{m s} \mathbf{s}^{(-\mathbf{1})}\right.$ & $\boldsymbol{d}_{\boldsymbol{o}}(\mathbf{m})$ & $\mathbf{R e}_{\text {equiv }}$ \\
\hline 2 & 0.05 & 0.4 & 0.09 & 9824.19 \\
4 & 0.05 & 0.8 & 0.09 & 19648.38 \\
6 & 0.05 & 1.2 & 0.09 & 29472.56 \\
8 & 0.05 & 1.6 & 0.09 & 39296.75 \\
10 & 0.05 & 2.0 & 0.09 & 49120.94
\end{tabular}

\section{3. Solution procedure}

The axisymmetric turbulent coaxial jet flow field was calculated by solving the Reynolds Averaged Navier-Stokes Equation (RANS) and turbulence models in ANSYS FLUENT (14.0) software. The equations were discretized by finite volume method and convective terms discretized using the quadratic upstream interpolation for convective kinetics (QUICK) scheme of Leonard (1979). The pressure-velocity coupling algorithm used was the Pressure Implicit of Splitting Operators (PISO) of [7]. Solutions were considered converged when the maximum tolerance of all the discretized equations was lower than $1 \times 10^{6}$, and when the total momentum in $z$-direction remain constant and independent of the distance $z$ from the nozzle.

The created mesh for the solution domain used for all computations in the present study is shown in Fig. 2.

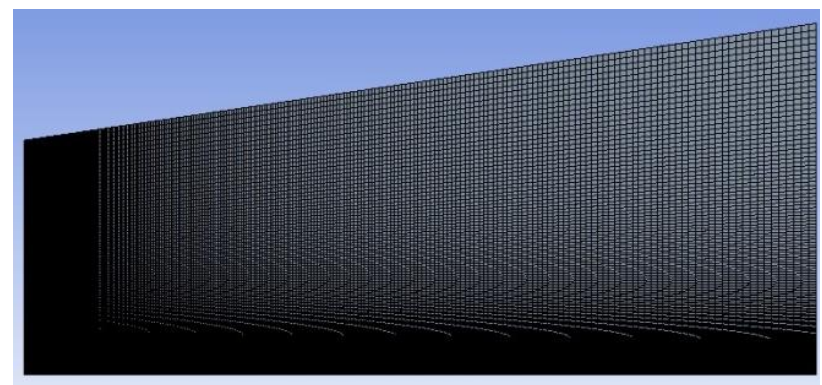

Fig. 2. Detail view of the grid for the solution domain

\section{Results and discussion}

The governing equation has been solved with ANSYS Fluent 14.0 United States Inc., a finite volume based software and the result presented in terms of decay of the centerline velocity, the turbulent kinetic energy, radial profile at different locations downstream of the flow field and the similarity profile for the varying Reynolds number. 


\section{1. Verification of Results}

The axial velocity profile of the coaxial jet centerline from the numerical analysis $(\operatorname{Re}=9824$, $\lambda=5$ ) is compared to those obtained from the experimental work of [2] with velocity ratio, $\lambda=4.54$ and Reynolds number of 7561. The comparison is presented in Fig. 3. The centreline velocity profile of the simulation has an agreeable curve to that of [2] which indicates that the numerical simulation performs reasonably well in estimating the decay. The average deviation between both results is due to the difference in the velocity ratio and Reynolds number.

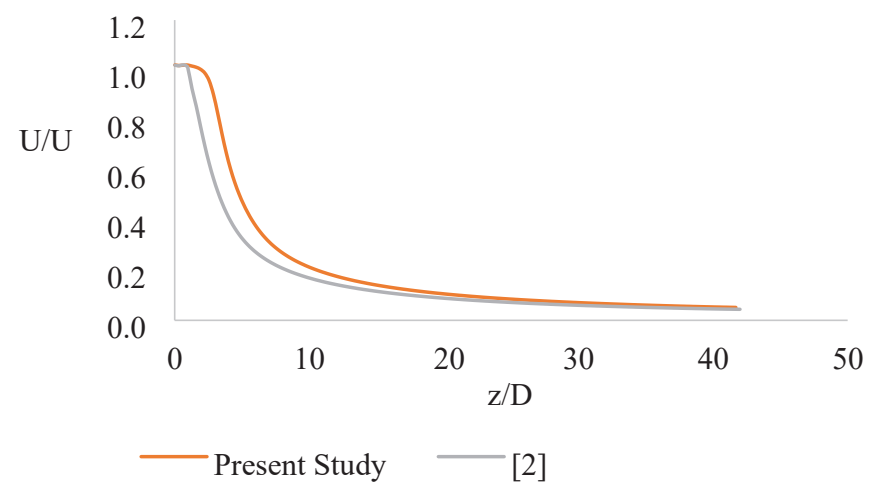

Fig. 3. Comparison of Numerical centerline velocities with the experimental work of [2]

\section{2. Stream Function Plot}

The Stream function (i.e. constant values along streamlines which are tangent to flow

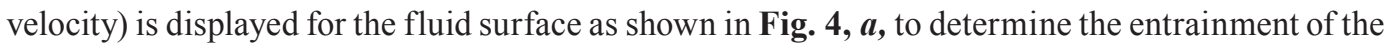
ambient air into the turbulent region. Fig. $\mathbf{4}, \boldsymbol{b}$ shows the Stream function for the fluid surface after the converged solution. Since the flow velocity is related to the stream function $\psi$ by $U=\partial \psi / \partial x$ and $V=-\partial \psi / \partial y$, the stream function is small where the velocity is high and vice - versa. From the contour plot, it is noticed that the velocity is high at the jet exit and decreases downstream due to the rapid mixing of the inner and outer jet and the surrounding fluid. Hence, the stream function will be smaller in the entrainment and jet stream areas because the flow is more strongly linear there and this is evident in the Fig. $\mathbf{4 ,} \boldsymbol{b}$.

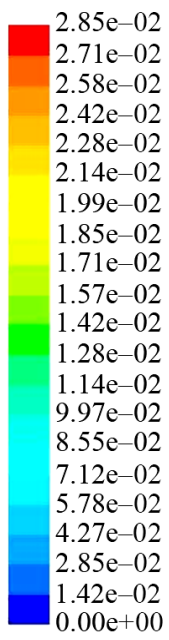

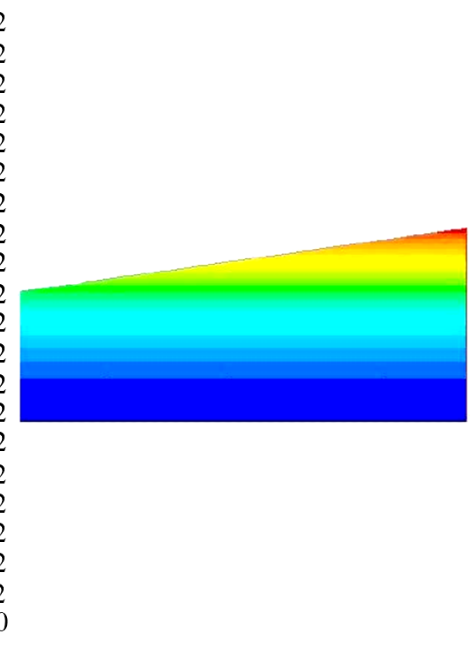

$a$

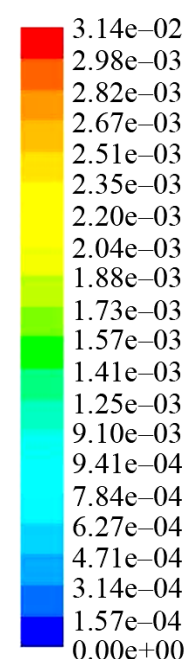

$0.00 \mathrm{e}+00$

$41 \mathrm{e}-04$

$84 \mathrm{e}-04$

$6.27 \mathrm{e}-04$

.71e-04

$14 \mathrm{e}-04$

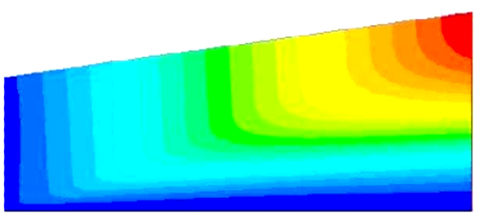

Fig. 4. Stream function: $a$ - on initialization of the Solutions; $b$ - the Converged Solution

\section{3. Centreline Velocity Plot}

Fig. 5 shows the decay of the axial mean velocities along the jet centerline for the varying Reynolds number. The coordinate $z$ denotes the downstream distance from the jet origin with $z=0$. 
The velocity profile is normalized by the mean velocity at the jet exit. The mean velocity plot shows a consistent trend for all the investigated Reynolds number. It could be seen that the potential core length is larger for the flow with lower Reynolds number due to the fact that the mean momentum rate of the jet with the lower Reynolds number is lower than with the jet with high Reynolds number. This is in consonance with the assertion of [1] that the potential core length of the flow will be more if the jet decays at a slower rate.

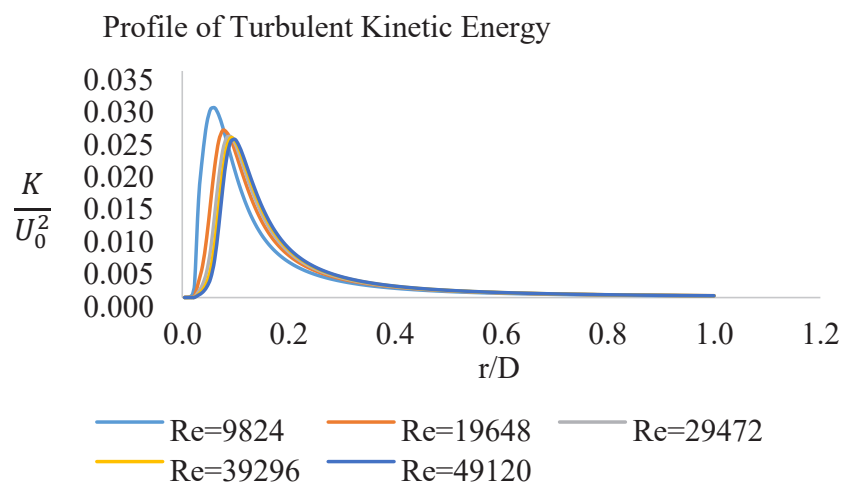

Fig. 5. Centreline Velocity Decay for various Reynolds numbers

\section{4. Turbulent Kinetic Energy Plot}

The turbulent kinetic energy is the mean kinetic energy per unit mass in the fluctuating velocity field. From the $k$-equation shown in Table 1, it is seen that the turbulent kinetic energy is governed by the production term $P_{k}$, a dissipation term $\varepsilon$, mean flow convection $\partial k / \partial t$ and turbulent transport. It worth noting (Fig. 6) that just after the inlet there is a peak in the turbulent kinetic energy due to the dominant production term $P_{k}$. It then approaches zero with increase in the distance as the fluid is convected and dissipated away from the centerline into the surrounding. Similarly, at the jet exit, the flow with the lowest Reynolds number have the highest turbulent kinetic energy because it suffers the greater shear, as the flow moves downstream, the turbulent kinetic energy decreases until far from the nozzle the jet presents the smallest value of turbulent kinetic energy.

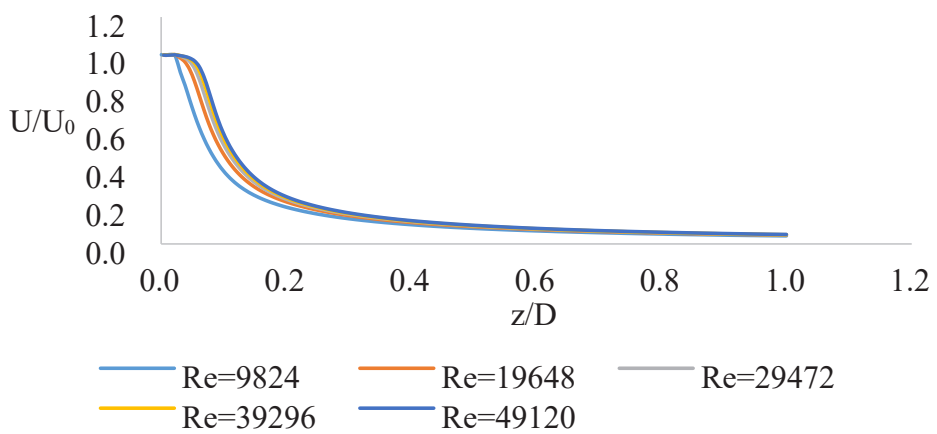

Fig. 6. Turbulent Kinetic Energy Profile for various Reynolds number

\section{5. Radial Profiles of the Axial mean Velocity}

In order to inspect the progression of flow of the different Reynolds number, the radial profiles of the axial mean velocity are conveyed, Fig. 7, $\boldsymbol{a}-\boldsymbol{e}$. Each subfigure represents the velocity profiles for the locations $z / D=10,25,40,55$ and 70 respectively. Within each figure, velocity profiles are displayed for the Reynolds numbers of 9824, 19648, 29472, 39296 and 49120. As stated earlier the velocity profiles are normalized by the mean axial velocity at the jet exit. By visual inspection of the figures, it appears that the trend display in the figures 
is consistent with the different locations. However, the mean decay velocity is greater for the highest Reynolds number and as expected the mean velocity for the highest Reynolds number is larger than for the others.

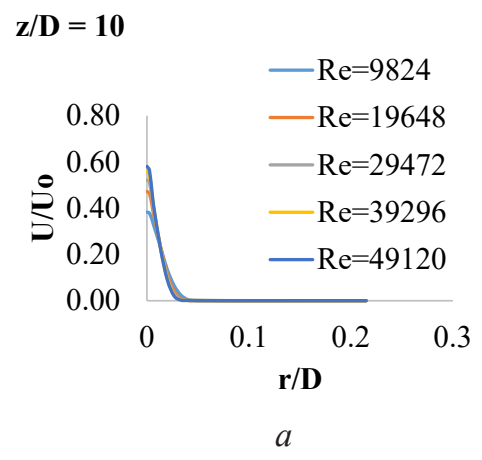

$\mathbf{Z} / \mathbf{D}=\mathbf{5 5}$

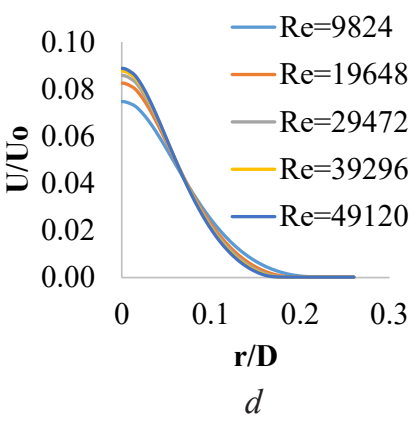

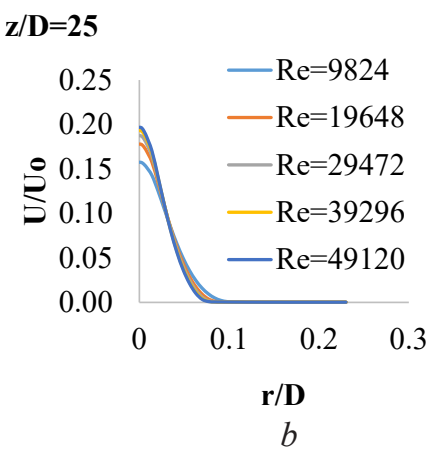

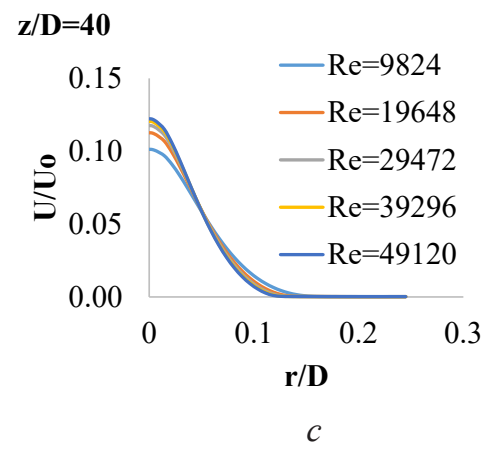

$\mathbf{z} / \mathbf{D}=\mathbf{7 0}$

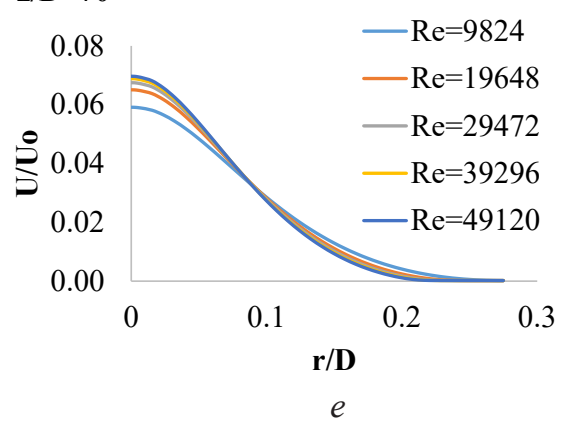

Fig. 7. Radial Profiles of the Axial mean Velocity of Coaxial jet for: $a-z / D=10 ; b-z / D=25$;

$$
c-z / D=40 ; d-z / D=55 ; e-z / D=70
$$

\section{6. Similarity Profiles}

Fig. 8, $\boldsymbol{a}-\boldsymbol{e}$ shows the plot of the self-similarity profile of the turbulent axial velocity for the different Reynolds numbers considered. Within each figure, self-similarity plot are displayed for the locations $z / D=10,25,40,55$ and 70 . The profile is similar to those in [8,9]. The figure indicates the tendency of the coaxial jet to behave like a single jet at some distance downstream of the end of the potential core and eventually attain self-preservation. Self-preservation occurs when the profile of any quantity (e. g. velocity) is brought into congruence by simple scale factors which depend only on one of the variables. A consequence of self-preservation is that the dynamical equations become independent of that variable, and are reduced by one variable in their functional dependence [10].

In cylindrical coordinates, the self - similar radial profile of the mean velocity can be expressed as shown in (3):

$$
\frac{u}{u_{0}}=\exp \left(-0.693 \eta^{2}\right) \text { and } \eta=\frac{r}{r_{1 / 2}},
$$

$r_{1 / 2}$ is the half radius/width, which is a measure of the jet growth rate and is defined as the radial location at which the local mean scalar is equal to half its value at the centerline, i.e. $u_{r_{1 / 2}}=0.5 u_{r}$. The expression on the right-hand side of the equation accounts for all of the radial variation and their existence implies that the profile of the velocity at every downstream location can be collapsed into single curves [2].

For all the Reynolds number considered, it is observed that at about a distance of $25 \mathrm{D}$, the similarity profile collapse into a single curve modeling the nature of a single jet. The similarity is however not confirmed in the developing region. 


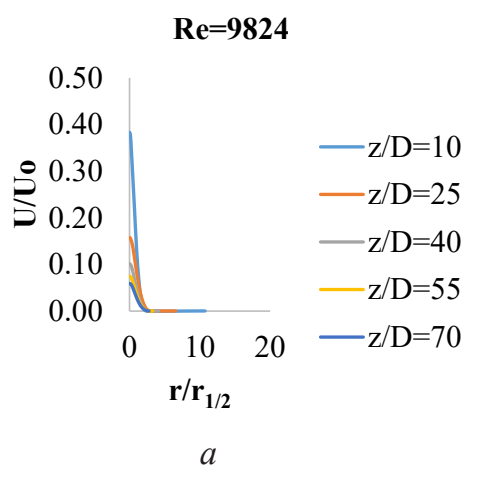

$\operatorname{Re}=39296$

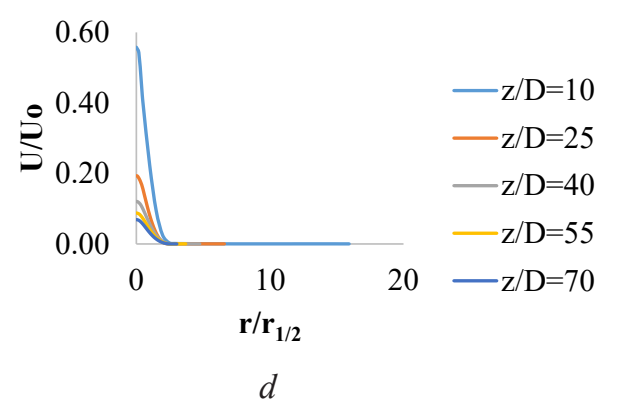

$\operatorname{Re}=19648$

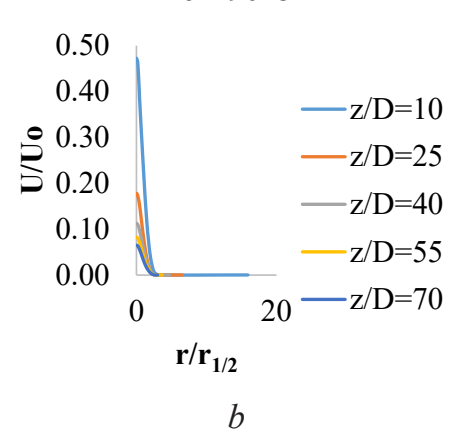

$\operatorname{Re}=\mathbf{2 9 4 7 2}$

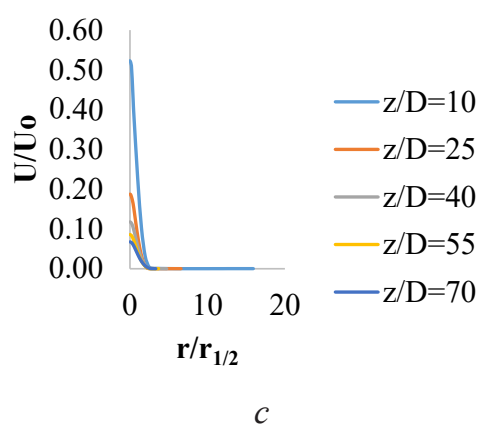

$\operatorname{Re}=49120$

Fig. 8. Similarity Profile for: $a-\operatorname{Re}=9824 ; b-\operatorname{Re}=19648 ; c-\operatorname{Re}=29472$;

$$
d-\operatorname{Re}=39296 ; e-\operatorname{Re}=49120
$$

It should be noted that this study is limited in scope to isothermal turbulent coaxial free jet over a range of Reynolds numbers in two-dimensional.

However, future studies should explore non-isothermal and time-dependent turbulent flow at extremely high Reynolds numbers in three-dimensional. The advantage of this present study is that the range of Reynolds numbers considered is of interest in engineering applications. It gives clear indication of the phenomenon so as to make provision for control if necessary. Experimental modeling should also be considered to authenticate the findings of the numerical modelling.

\section{Conclusions}

The effect of high Reynolds number on axisymmetric free incompressible isothermal turbulent coaxial free jet has been examined using a finite volume based Computational Fluid Dynamics (CFD), ANSYS Fluent for various Reynolds numbers.

The results that stemmed out can be summarized as following:

- the decay of the axial mean velocities along the jet centerline and the radial profiles of the mean and fluctuating velocities depend on the Reynolds number;

- the flow with higher Reynolds number has a shorter potential core thereby increasing turbulence and enhancing mixing than those with low Reynolds number;

- the turbulent kinetic energy profile depends on the Reynolds number;

- the coaxial jet flow showed self-similarity at about $z / D=25$;

- the spreading of the jet was more or less independent of the Reynolds number beyond the self-similarity region.

\section{References}

[1] Kathiresan, K., Balamani, A., Karthik, M., Gogulanathan, P., Thanikaivel, D. M., Ilakkiya, S. (2014). CFD Analysis of Supersonic Coaxial Jets on Effect of Spreading Rates. Journal of Engineering Research and Applications, 4, 29-35.

[2] Ströher, G. R., Martins, C. A., De Andrade, C. R. (2010). Numerical and experimental study of a free incompressible isothermal turbulent coaxial jet. Revista de Engenharia Térmica, 9 (1-2), 98. doi: https://doi.org/10.5380/reterm.v9i1-2.61939 
[3] Villermaux, E., Rehab, H. (2000). Mixing in coaxial jets. Journal of Fluid Mechanics, 425, 161-185. doi: https://doi.org/10.1017/ s002211200000210x

[4] Celik, N., Bettenhausen, D. W., Lovik, R. D. (2012). Formation of Co-axial jets and their Downstream Development. Journal of Thermal Science and Technology, 32 (1), 91-99.

[5] Ranga Dinesh, K. K. J., Savill, A. M., Jenkins, K. W., Kirkpatrick, M. P. (2009). A study of mixing and intermittency in a coaxial turbulent jet. Fluid Dynamics Research, 42 (2), 025507. doi: https://doi.org/10.1088/0169-5983/42/2/025507

[6] Shih, T.-H., Liou, W. W., Shabbir, A., Yang, Z., Zhu, J. (1995). A new $k-\varepsilon$ eddy viscosity model for high Reynolds number turbulent flows. Computers \& Fluids, 24 (3), 227-238. doi: https://doi.org/10.1016/0045-7930(94)00032-t

[7] Issa, R. I. (1986). Solution of the implicitly discretised fluid flow equations by operator-splitting. Journal of Computational Physics, 62 (1), 40-65. doi: https://doi.org/10.1016/0021-9991(86)90099-9

[8] Abramovich, G. N.; Schindel, L. (Ed.) (2003). The Theory of Turbulent Jets. The MIT Press. doi: https://doi.org/10.7551/ mitpress/6781.001.0001

[9] Schlichting, H. (1979). Boundary-Layer Theory. McGraw-Hill. Available at: http://ae.sharif.edu/ viscousflow/Schlichting \%20-\%20Boundary\%20Layer\%20Theory.pdf

[10] Pope, S. B. (2000). Turbulent Flows. Cambridge University Press, 802. doi: https://doi.org/10.1017/cbo9780511840531

How to cite: Oyewola, O. M., Ismail, O. S., Sanni, L. A. (2022). Numerical simulation of reynolds number effect of a free incompressible isothermal turbulent coaxial jet. EUREKA: Physics and Engineering, 1, 3-11. doi: https://doi.org/10.21303/ 2461-4262.2022.001896 